\title{
Blaving comme TICE pour ameliorer la fluidite des apprenants de français*
}

\author{
Derly Cervantes Cerra ${ }^{1}$ \\ Universidad del Atlántico, Barranquilla, Colombia
}

DOI: http://dx.doi.org/10.17081/eduhum.18.30.1323

Recibido: 30 de marzo de 2015

Aceptado: 21 de agosto de 2015

\section{Blaving as ICT Tool to improve students' fluency in the french language}

Mots-clés:
Blaving, Fluidité, Production orale,
TICE, Amélioration.

Palabras clave:

Blaving, Fluidez, Producción oral, TICE, Mejoramiento.

Key words:

Blaving, Fluency, Speaking performance, ICT, Language development.

\begin{abstract}
Résumé
L'objectif de cette recherche a été de comparer le niveau de fluidité des étudiants de français de l'Universidad del Atlántico, avant et après avoir utilisé le réseau social Blaving. Pour mener à bien cette comparaison, nous avons fait des interviews, des enquêtes et des enregistrements en vue d' une activité diagnostique qui consistait à répondre oralement à une question en espagnol et à une autre en français, en une minute. Dans cette activité-là, en premier lieu, on a vu reflété leur manque de fluidité et leurs erreurs de prononciation. Cependant tout de suite avec l'aide du réseau social Blaving, ils ont considérablement amélioré dans ces aspects linguistiques. Autrement dit, les résultats obtenus ont été l'augmentation du nombre de syllabes prononcées en français pendant une minute et l'amélioration de leur prononciation.
\end{abstract}

\section{Resumen}

El objetivo de la presente investigación consistió en comparar el nivel de fluidez de los estudiantes de francés de la Universidad del Atlántico, antes y después de utilizar la red social Blaving. Para llevar a cabo esta comparación, realizamos entrevistas, encuestas y grabaciones enfocadas hacia una actividad diagnóstica que consistía en responder oralmente una pregunta en español y otra en francés durante un minuto. En dicha actividad, se reflejó, en primera instancia, su falta de fluidez y sus errores de pronunciación. Pero, luego, con el apoyo de la red social Blaving, los estudiantes mejoraron sustancialmente en estos aspectos lingüísticos. En otras palabras, los resultados obtenidos fueron dos: el aumento del número de sílabas pronunciadas en francés durante un minuto y el mejoramiento de su pronunciación.

\begin{abstract}
The purpose of this research study was to compare the level of fluency and pronunciation among students of French at the Universidad del Atlántico before and after the use of the social network Blaving. In order to carry out this comparison, a series of interviews and surveys were administered and an assessment of the students' pronunciation was made through the recording of their one-minute answers to a question in French and Spanish. In this diagnostic phase, students evidenced both fluency and pronunciation problems in French. These linguistic drawbacks, nonetheless, were considerably reduced with the help of the activities implemented in Blaving. Overall, findings showed an increase in the number of syllables pronounced by the learners in one minute and some improvement in the pronunciation of the target language.
\end{abstract}

\section{(ब) $\Theta \Theta$}

Referencia de este artículo (APA): Cervantes, D. (2016). Blaving comme TICE pour ameliorer la fluidite des apprenants de français. En Revista Educación y Humanismo, 18(30), 71-91. http://dx.doi.org/10.17081/eduhum.18.30.1323

* Este artículo es producto de la tesis del Master 2 en Francés Lengua Extranjera sobre el uso de la red social Blaving para mejorar la fluidez de los estudiantes de francés.

1. Licenciada en Idiomas Extranjeros. Universidad del Atlántico, Barranquilla, Colombia. Magíster en Francés Lengua Extranjera. Universidad de Martinica, Francia. Profesora de Francés de la Universidad del Atlántico (Colombia). Master en FLE.

Email: derlycervantes@hotmail.com 


\section{Introduction}

Ce qui a motivé cette recherche c'est que les élèves veulent acquérir une compétence communicative fluide dans la production orale. Cela est dû au fait de vouloir un contact direct avec les francophones. Le manque d'activités pour mettre en pratique les connaissances et les habiletés communicatives des élèves, c'est l'une des difficultés rencontrées dans les cours. Celles-ci sont faciles à distinguer à partir de son observation et son développement. Compte tenu de ces variables qui influencent sur le plein développement de l'acquisition du français dans notre contexte, il est nécessaire de faire l'analyse des différents aspects qui limitent cet apprentissage. Pour cette raison, ce sont les principales difficultés observées dans le cours de français de huitième semestre de Licence en Langues Étrangères de 1'Universidad del Atlántico, par exemple: la peur de commettre des erreurs, la crainte de s'exprimer en public, le manque de vocabulaire, l'apprenant ne sait pas quoi dire et comment le dire, le manque de pratique et d'activités de production orale, le manque d'un laboratoire de langues spécialisé (il faut accorder langues et spécialisé).

Comme ces limitations nous mène à chercher un outil qui nous permet d'améliorer les erreurs et les difficultés des apprenants, nous avons choisi l'usage du réseau social Blaving qui nous aidera à atteindre l'objectif principal de ce mémoire.

D'autre part, le développement des réseaux de communication pratiquement instantanée ainsi que la nature de la langue elle-même ont fait de la communication orale une forme d'échange privilégiée. La décision d'apprendre une langue étrangère évoque plus profondément l'idée d'apprendre à la parler que d'apprendre à l'écrire. Un élève arrive à maîtriser la production orale à travers la pratique de manière permanente.

Dans ce mémoire, nous parlerons des concepts de fluidité, production orale, prononciation et du réseau social Blaving que nous avons employé pour améliorer la fluidité et la production orale des étudiants du cours de français de huitième semestre de Licence en Langues Étrangères de l’Universidad del Atlántico.

\section{Objectif général}

Atteindre une plus grande fluidité en expression orale ainsi qu'une meilleure prononciation des élèves de huitième semestre du programme de Licence en Langues Étrangères de l'Universidad del Atlántico.

\section{Objectifs spécifiques}

Obtenir une meilleure fluidité dans le débit à l'oral en utilisant le réseau social Blaving.

Améliorer la prononciation en développant des activités proposées dans ce réseau.

\section{Cadre théorique}

Nous trouvons que la production orale est importante puisque c'est la manière de communication essentielle du genre humain. Si nos élèves 
vont enseigner le français, ils doivent avoir une bonne maîtrise de la langue parlée. S'ils n'osent pas prononcer un mot en français, comment feront-ils pour expliquer leurs cours? Ce serait une frustration. Nous, en tant qu'enseignants, devons chercher la manière d'améliorer la performance de nos apprenants dans la production orale.

De nos jours, il y a beaucoup d'élèves qui trouvent des difficultés à l'étranger parce qu'ils ne se sentent pas bien formés pour parler la langue du pays. C'est un problème très grave. Nous devons surmonter la peur de nous exprimer oralement. En plus, si nos apprenants maîtrisent de manière convenable la communication orale en français, ils auront de meilleures possibilités de communiquer avec les francophones. L'objectif principal de l'enseignement des langues, c'est amener les élèves à communiquer dans une langue étrangère.

\section{La Fluidité, la Production Orale et la Pho-} nétique

Comme le thème de ce mémoire est l'amélioration de la fluidité verbale en production orale, il est essentiel d'aborder les définitions de fluidité, production orale et phonétique.

\section{Définition de Fluidité}

La fluidité fait référence à la qualité et au nombre de mots prononcés. C'est «le nombre de mots ou de phrases (généralement, dans un discours spontané ou lu) que le sujet est capable de produire en un temps donné, et éventuellement selon une consigne ou une situation don- née» (Glossaire Universel de la Psychologie et des Sciences Cognitives, 2011) «Elle se rapporte aussi à la qualité de l'information émise puisqu'il n'est pas suffisant de produire beaucoup de mots par minute mais aussi de produire un discours qui montre un développement et qui s'oriente vers un but discursif» (Menjura, 2007, p.8).

\section{La Production Orale}

La langue orale est synonyme de la langue parlée. «L'oral est un fait de vive voix, transmis par la voix» (Le Petit Larousse illustré, 1995). Notre voix est la matière première de la communication orale et peut être notre meilleur outil.

La production orale est une compétence que les apprenants doivent progressivement acquérir, qui consiste à s'exprimer dans les situations les plus diverses. Les élèves doivent être capables de s'exprimer dans la langue étrangère dans toutes les situations de la vie quotidienne.

\section{Phonétique}

La phonétique est la science qui étudie les sons du langage. D'après nous, la phonétique est primordiale dans l'enseignement des langues étrangères. Dans le cas de la prononciation du français, il paraît important de bien prononcer les mots puisque si on prononce mal, le message ne va pas être compris de la même manière.

Il faut connaître les traits essentiels de la phonétique du français, les caractéristiques des phonèmes et un certain nombre de règles concernait la manière dont sont transcrits ces 
phonèmes. Mais il faut aussi s'entraîner à répéter les phonèmes: la prononciation est un exercice physique, il s'agit de de prononcer les phonèmes différents de ceux auxquels on est habitué. Par exemple, pour les élèves hispanophones il faut leur faire pratiquer les voyelles nasales, les lettres: [J], [3], [в], [v], [ø], [y], [z], parce que ces sons n'existent pas en espagnol. Donc, ils ne sont pas habitués à les prononcer dans leur langue maternelle.

\section{Problématique}

Dans ce mémoire nous nous posons les questions suivantes:

Comment améliorer la fluidité en production orale en FLE des élèves (adolescents) de huitième semestre de Licence en Langues Étrangères de l'Universidad del Atlántico (Colombie) grâce au réseau social Blaving?

Comment développer la fluidité en production orale des élèves en quantité et en qualité?

Quelles sont les avantages du réseau social Blaving?

Comment développer l'aisance en production orale chez les élèves?

J'enseigne le français à l'Universidad del Atlántico (Colombie) aux étudiants de huitième semestre de Licence en Langues Étrangères. Ma classe de cette année est composée par 16 étudiants. L'âge moyen de mes étudiants est 18 ans. Ils ont 4 heures de français par semaine (80 heures par semestre). Le manuel qu'on utilise dans le cours de français est Écho B1 volume 2. M. Stirman/ J. Girardet, CLE International, Paris, 2013.

Tout au long de mon expérience universitaire nous nous sommes rendu compte que le problème plus remarquable chez les apprenants, c'est la difficulté de communiquer avec fluidité. Celle-ci est en raison de la peur de commettre des erreurs, la crainte de s'exprimer en public, le manque de vocabulaire, le fait de ne pas savoir quoi dire et comment le dire et le manque de pratique et d'activités de production orale.

Ensuite, nous allons décrire ces difficultés de manière détaillée:

\section{La peur de commettre des erreurs}

Dans une perspective d'un enseignement centré sur l'apprenant, les notions d'apprentissage et d'erreur occupent actuellement en didactique de langues, une place centrale.

Il existe grosso modo deux attitudes vis-à-vis des erreurs des apprenants. Selon la première, leur apparition est le signe d'un enseignement inadéquat, avec une méthode et un enseignement parfait, il n'y aurait jamais d'erreurs. D'après, la seconde, il est inévitable que des erreurs apparaissent malgré tous nos efforts. Il faudra toujours s'y attendre et se résigner à les traiter lors qu'elles apparaissent. Il existe cependant une troisième attitude qui voit dans 
l'erreur, une manifestation naturelle et nécessaire des processus d'apprentissage pour cette dernière, les erreurs ont une triple signification (Corder, 1980, p.13).

Il est important que nous remarquons la définition suivante du mot faute:

Conséquence de la non-conformité à ce qui est standard ou concret. La notion de faute embrasse des faites différents selon que le standard est d'ordre normatif ou descriptif. En termes normatifs, elle qualifiera des usages non standards, c'est en ce sens qu'il faut entendre fautes d'orthographes. En termes descriptifs, la faute qualifie la non adéquation aux règles ou aux schémas de la langue (Arrivé, Gadet \& Galmiche, 1986, p.286).

Je suis d'accord avec cette définition d'erreur:

L'erreur n'est que la manifestation de ce que l'on appelle l'interlangue, c'est-à-dire l'état de maîtrise provisoire de la langue étrangère en train de se former. L'erreur fait partie de la langue intégrante de cette langue intermédiaire entre les balbutiements du départ et l'état de maîtrise relative final. C'est donc par ses erreurs que testent ses hypothèses de fonctionnement du système nouveau qu'il est en train de se créer (Tagliante, 1994, p.40).

Selon la définition de Tagliante, l'apprenant a la capacité de construire son propre savoir par et grâce à ses erreurs.
Il est important d'esquisser cette définition d'interlangue:

La connaissance et l'utilisation non natives d'une langue quelconque par un sujet non natif et non-équilingue, c'est-à-dire un système autre que celui de la langue cible mais qui, a quelque stade d'apprentissage qu'on l'appréhende, en convoite certaines composantes, c'est ce que nous avons précédemment appelé grammaire intériorisée par l'apprenant et que nous appelons également ici: interlangue (Besse \& Porquier, 1991, p.217).

\section{Nous signalons aussi que:}

La langue qui se forme chez un apprenant d'une langue étrangère à mesure qu'il est confronté à des éléments de la langue cible, sans pour autant qu'elle coïncide totalement avec cette langue cible. Dans la constitution de l'interlangue entrent la langue maternelle éventuellement d'autres langues étrangères préalablement acquises, et la langue cible, son impact, son stade de développement, ses aspects idiosyncratiques dépendent notamment de variables individuelles, sociales, en rapport avec la situation d'apprentissage ainsi que, le cas échéant des variables didactiques méthodologiques (Vogel, 1995, p.20).

Selon ces deux concepts, l'interlangue est une stratégie d'apprentissage utilisée par l'élève pour résoudre ses difficultés d'apprentissage chaque apprenant possède sa propre interlangue. 
L'interférence produit des transferts négatifs des structures des langues maternelles et langues secondes préalablement acquises sur la langue cible. L'enseignant peut facilement repérer l'interférence s'il connaît la langue pratiquée par l'apprenant.

\section{La crainte de s'exprimer en public}

L'orateur doit être sûr de son succès avoir une pensée positive.

Il faut s'approprier le sujet: on doit être capable de donner des exemples personnels, de donner notre point de vue et de parler de l'humain (émotions, sentiments, etc.).

Il ne faut pas apprendre par cœur pour obtenir un discours fluide et naturel. On doit parler comme dans une conversation normale et laisser transparaître ses émotions.

Les buts de l'intervention orale doivent être: convaincre, faire agir, informer et distraire (Carnegie, 2014, p.4).

Pour de nombreuses personnes, la peur de parler en public constitue une incapacité qui peut être un obstacle à une promotion, au bon déroulement de leur carrière, etc.

\section{Le manque de vocabulaire}

Un autre problème auquel les apprenants sont confrontés, c'est le manque d'un vocabulaire adéquat. L'expérience la plus frustrante pour les étudiants de langues étrangères, c'est de ne pas être capables de trouver le mot précis pour exprimer leurs propres idées et sentiments. C'est pourquoi ils ont besoin d'enrichir leur vocabulaire. Le professeur de langues doit mettre en pratique des activités réutilisant le vocabulaire appris.

\section{L'apprenant ne sait pas quoi dire et com-} ment le dire

Nous avons remarqué que quand on demande aux étudiants de donner leur point de vue sur un thème, ils mettent beaucoup de temps à s'exprimer. Cela est dû au fait qu'ils ont beaucoup d'idées, cependant ils ne sont pas capables de les organiser de manière cohérente. Pour cette raison, ils ont du mal à faire une analyse d'un texte et exprimer leurs idées.

La première question qu'on doit se poser avant de prendre la parole en public est: Quel est l'objectif de mon intervention? Est-ce pour informer? Motiver? Divertir? Convaincre? Notre objectif ne sera pas le même selon que nous intervenions pour un discours, un exposé, un débat, etc.

Si une personne ne sait pas clairement pourquoi elle va intervenir, il est certain qu'elle va se planter. Alors, il faut qu'elle réfléchisse bien à cette simple question.

Il est nécessaire de bien définir son message: Quelle est l'idée principale qu'on veut faire passer? 
Un exercice qui peut nous aider à structurer notre pensée, c'est nous poser la question suivante: Si je devais résumer mon intervention en une seule phrase, quelle serait-elle?

\section{Le manque de pratique et d'activités de production orale}

Il faut que les élèves s'habituent plus souvent à l'oral à la langue étrangère à travers différentes activités, telles que: exposés, débats, tables rondes, jeux de rôles, etc. Plus les élèves parlent plus ils pratiquent l'oral.

\section{Justification}

Comme nous sommes immergés dans un processus de globalisation, notre pays a des relations commerciales, sociales et diplomatiques avec d'autres pays. Pour cela, il est nécessaire que nous maîtrisions plusieurs langues, parmi celles-ci, le français. Si nous maîtrisons mieux une langue étrangère, la possibilité de relations avec le pays étranger augmente. La France, c'est l'une des nations avec laquelle la Colombie maintient des relations étroites politiques et commerciales. Par conséquent, l'apprentissage du français est important pour les Colombiens.

\section{Les Nouvelles Technologies de L'informa- tion et de la Communication (NTIC)}

Les technologies sont souvent utilisées et comprises comme synonymes d'internet avec ce que tout cela suppose: l'utilisation d'ordinateurs et de divers réseaux et de communications permettant de relier les utilisateurs entre eux, et de les relier à l'information. En effet, les tech- nologies qui supportent et permettent le développement d'internet sont aujourd'hui au cœur des NTIC mais ces dernières ne se limitent pas à l'internet. Les NTIC doit être ici entendu comme recouvrant l'ensemble de la convergence des télécommunications, de l'information et de l'audiovisuel comme dénominateur commun l'utilisation de données numériques. Ministère de la Communication Niger (2003).

Actuellement, les nouvelles technologies ont atteint un très grand développement dans presque tous les domaines, surtout dans le domaine éducatif. Ils deviennent une nécessité dans le contexte de la société où les changements rapides, l'augmentation de connaissances et les domaines d'une éducation de haut niveau constamment mise à jour se transforment en une exigence permanente (Rosario, 2005).

Grâce aux NTIC on a eu des changements très importants du monde entier, on peut améliorer les capacités créatives, l'imagination, les capacités communicatives et le vocabulaire qui permettent d'accéder à une plus grande quantité d'informations et qui fournissent les moyens pour atteindre un meilleur développement intégral des êtres humains. Tous les établissements d'enseignement doivent disposer de matériel informatique qui permet aux élèves d'avoir accès à internet.

Nous, en tant qu'enseignants, devons recourir aux réseaux sociaux pour changer la manière dont les personnes interagissent et se mettent 
en relation afin d'améliorer leurs compétences communicatives en français.

\section{Réseau social basé sur la voix: Blaving}

Le réseau social Blaving (www.blaving. com) a été créé en mai 2011 par l'entreprise argentine-brésilienne Pmovil pour que les usagers interagissent avec d'autres personnes. Ce réseau est disponible en trois langues: anglais, espagnol, et portugais. Nous avons le droit à enregistrer un message d'une durée de deux minutes. En plus, nous pouvons relier ce service à twitter, facebook, orkut, myspace pour qu'il puisse envoyer le message systématiquement sur ces réseaux sociaux. Nous avons la possibilité de télécharger une application pour ces smartphones. Ce qu'il faut avoir pour tous les services offerts sur internet.

Blaving fonctionne comme n'importe quel autre réseau, la différence est qu'on peut enregistrer les messages directement ou si on préfère on peut également télécharger un fichier audio qu'on peut même ajouter une photo. Chaque message peut être accompagné d'un titre, le lieu où il a été enregistré, les étiquettes et une image d'identification. Nous pouvons également télécharger à partir de l'iphone, ipod, android, blackberry, et java.

Blaving est un service gratuit. C'est dont vous avez besoin, c'est de vous inscrire et on peut l'utiliser à partir de son téléphone mobile. Il est idéal pour ceux qui ne veulent pas communiquer par messages de texte s'ils sont dans la rue.

\section{Comment fonctionne le réseau Blaving?}

D'abord, nous devons nous inscrire comme nouvel utilisateur et nous diriger vers login pour commencer à télécharger des messages audio, appelés Blavs d'une durée de 2 minutes, Si on ne veut pas parler et on préfère télécharger un sujet, on peut aussi le faire depuis l'option: télécharger le ficher existant. Si on choisit enregistrer un Blav de voix, on va trouver un mini-graveur avec une aiguille qui indique les minutes et un mesureur de fréquences de son. Ensuite, le graveur traite l'information et nous demande d'ajouter l'information au Blav pour l'étiqueter. Puis, le Blav apparaît publié dans notre profil.

Ce réseau est très utile dans la salle de classe parce qu'il rend possible la création de forums à l'oral de manière simple et rapide. Il aide à développer un apprentissage actif qui conduit l'élève à l'action et aussi un apprentissage programmatique parce qu'il faut aussi comprendre l'action. Il nous aide à développer les habiletés de la pensée telles que: la créativité, la synthèse, la déduction, le travail en équipe, l'organisation, l'évaluation, l'analyse, la clarification, la sélection et la structuration de l'information. Il est adéquat pour créer des forums et des débats en ligne avec des opinions, des présentations personnelles, des messages, etc.

Avec Blaving les étudiants peuvent pratiquer la prononciation, en développant entre autres l'autoévaluation puisque chaque message reste enregistré sur le réseau pour un temps indéfini en permettant à l'étudiant de s'écouter parler, 
corriger ses erreurs, enregistrer un nouveau message. Encore une fois il s'écoute parler. Il répète ce processus jusqu'à ce qu'il perfectionne sa prononciation. Ce nouveau mécanisme permet au professeur d'identifier de manière détaillée les fautes de prononciation de chaque apprenant parce que les enregistrements sont toujours disponibles sur internet. Nous pourrions enregistrer des exemples avec la manière correcte de prononciation qui sert comme un guide et feedback permanent à l'étudiant.

Nous pouvons télécharger sur Blaving de différentes activités, par exemple: des exercices de discrimination auditive, des micros-trottoirs, des reportages, des entretiens, des flash d'information, etc. Puis, nous leur expliquons les consignes pour chaque activité et leur demande de les faire de manière consciencieuse.

\section{Méthodologie}

D'abord, nous avons fait une activité diagnostique, en demandant aux élèves de huitième semestre de Licence en Langues Étrangères de répondre oralement, en espagnol, à la question suivante: ¿Por qué le gusta el francés? Ils devaient parler pendant une minute en espagnol. Ensuite, nous leur avons demandé de comparer la Colombie et la France et leur réponse devait être en français et durer une minute. Bien sûr, nous avons enregistré tout ce qu'ils ont dit. Comme il fallait s'inscrire sur Blaving, nous leur avons demandé de le faire. Pour qu'ils comprennent mieux le processus, ils l'ont regardé sur ce lien: http://es.blaving.com/videos. Une fois qu'ils se seront inscrits sur Blaving, ils devront enregistrer leur premier message en français d'une minute sur n'importe quel thème. D'après nous, nous devons donner un bon exemple aux étudiants. C'est pourquoi nous avons enregistré un premier message sur Blaving. Nous avons aussi expliqué sur ce réseau quelles étaient les raisons pour apprendre le français. Cela stimule les élèves.

Après, nous avons écouté les enregistrements des deux questions que je leur avais posé, en comptant le nombre de syllabes qu'ils ont prononcé en espagnol et en français. Bien évidemment, ils ont prononcé plus de syllabes en espagnol qu'en français. Il me paraît pertinent de comparer le nombre de syllabes en français prononcées par un locuteur natif et mon étudiante (hispanophone) qui a prononcé plus de syllabes en français en une minute: le premier a prononcé 269 syllabes tandis que la deuxième a prononcé 152 syllabes. Parmi mes étudiants le moindre nombre de syllabes prononcées en français c'était 55. Ces résultats montrent que les apprenants n'ont pas émis assez de syllabes en une minute. Il faudrait qu'ils augmentent la quantité de syllabes par minute pour arriver à améliorer leur fluidité en production orale.

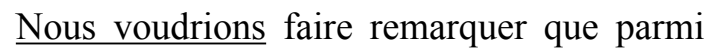
mes élèves il y a une qui a prononcé 237 syllabes en français. Son cas est différent parce qu'elle est restée en France du 6 octobre 2013 au 4 juillet 2014. Elle est partie avec le programme $A u$ Pair, de manière indépendante. En outre, elle a 
fait des études en FLE pendant 5 mois, à l'université de Toulouse-Le Mirail. Elle m'a dit que ce séjour a été très enrichissant parce qu'elle sent qu'elle a amélioré son niveau de langue.

Pour illustrer l'activité diagnostique dont nous avons parlé, nous avons élaboré un tableau qui indique le nombre de syllabes en français prononcées par chaque apprenant avec son pourcentage. En outre, j'ai fait un graphique qui contient le pourcentage du nombre de syllabes en français prononcées par les élèves.

En plus, nous avons relevé les fautes de prononciation en français les plus commises par les étudiants, telles que: la prononciation des lettres $[\varepsilon \mathrm{s}],[\mathrm{y}],[\tilde{\mathrm{a}}],[\tilde{\varepsilon}],[\tilde{\mathrm{o}}],[\mathrm{j}]$, les liaisons.

\section{Activité finale}

Comme il fallait vérifier la progression des apprenants, nous avons fait une activité finale dans laquelle ils devaient parler pendant une minute en français. Celle-ci consistait à répondre à la question suivante: Selon vous, quels sont les avantages et les inconvénients du réseau social Blaving?

Pour illustrer cette activité finale, nous avons élaboré un tableau qui indique le nombre de syllabes en français prononcées par chaque apprenant avec son pourcentage. En outre, nous avons fait un graphique qui contient le pourcentage d'amélioration du nombre de syllabes en français prononcées par les élèves.
Ce sont les résultats après avoir réalisé l'activité finale: Premièrement, tous les élèves ont augmenté le nombre de syllabes prononcées en français, c'est-à-dire, ils ont eu une augmentation dans la quantité de syllabes. Le plus haut nombre de syllabes c'était 239 tandis que le moindre nombre de syllabes c'était 133. En plus, ils ont amélioré leur prononciation.

\section{Conclusions}

\section{Les Réseaux Sociaux et l'Éducation}

Comme signalent Díaz \& Gómez (2013) les nouvelles technologies de l'information et de la communication dans l'enseignement ont produit une révolution selon ce qu'ils appellent le Web 2.0 , qui a permis la démocratisation de la toile.

Ces professeurs ont réalisé une étude de cas conduite dans un cours de FLE niveau avancé d'une université publique de la Colombie avec le but de connaître l'influence qu'a l'échange en ligne dans l'apprentissage du FLE. L'analyse de l'information a été faite sous la technique typologique dont les résultats présentent les principaux apports des applications qui offre ce web interactif et les réactions des étudiants lorsqu'ils les utilisent pour l'apprentissage d'une langue étrangère.

Selon elles, le Web 2.0 dans un contexte éducatif ou dans un cours de langue ont des avantages tant par les étudiants que par le professeur que l'on a contrasté avec l'analyse des observations. 
Les étudiants coïncident en affirmant que les réseaux sociaux donnent plus de possibilités à l'occasion d'élaborer des savoirs collectifs sans contrainte spatio-temporelle puisque cette application est la plus répandue.

Ils pensent que le web 2.0 est très intéressant, accessible et facile et pour cela il a une forte influence sur leur apprentissage et le développement de leurs devoirs.

Elles ont constaté que la télécollaboration a permis un développement significatif des activités avec l'utilisation des outils de communication en ligne variés, comme Facebook, la vidéo conférence, les blogs et EDMODO qui leur ont mis en contact avec des apprenants de langue de différents pays francophones dans le but de développer le travail collaboratif grâce à un intéressant échange interculturel.

Par ailleurs, les participants ont des perceptions positives par rapport à son utilisation, comme l'interaction avec la culture étrangère, l'importance de l'accompagnement du professeur dans et dehors la classe ainsi que le développement de compétences communicatives et linguistiques.

Finalement, ces chercheuses signalent que leur découverte globale c'est que ces applications web ont un potentiel énorme pour transformer beaucoup d'aspects de l'enseignement et l'apprentissage de langues quand les professeurs connaissent leur ample fonctionnement et son utilisation est accompagnée d'une planification rigoureuse et des activités significatives pour les étudiants. Une nouvelle génération de professeurs et d'étudiants utilise les outils du Web 2.0 pour créer des espaces virtuels qui supportent leurs buts pédagogiques et augmentent leur capacité éducative, étendant ainsi leur apprentissage au-delà des murs physiques de la salle de classe.

Dans notre recherche, après avoir mis en pratique l'usage du réseau social Blaving, nous pouvons assurer que cet outil a été fructueux dans le cours de français de huitième semestre de Licence en Langues Étrangères de l'Universidad del Atlántico.

D'abord, il y a eu une augmentation du nombre de syllabes en français prononcées par les apprenants en une minute.

Par rapport à la fluidité, nous trouvons que les élèves s'expriment avec plus de fluidité et d'aisance qu'avant. Quand nous leur avons fait l'activité diagnostique, ils avaient peur de parler parce qu'ils ne savaient pas quoi dire et comment le dire et ils craignaient de se tromper. Nous avons remarqué qu'ils sont plus sûrs à l'heure d'exprimer leurs idées en français. Ils ont plus de confiance en eux-mêmes.

En ce qui concerne la prononciation, ils ont amélioré la prononciation des liaisons et les sons suivants: $\mathrm{r}[\mathrm{R}], \mathrm{u}[\mathrm{y}]$, a nasal [ã], e nasal $[\tilde{\varepsilon}]$, o nasal [õ]. 


\section{Recommandations}

Nous trouvons que l'utilisation de ce réseau social a beaucoup apporté aux élèves parce qu'ils ont eu l'opportunité d'écouter leur propre voix et de cette manière se rendre compte de leurs propres erreurs et les corriger, d'écouter des locuteurs natifs pour remarquer la différence de prononciation entre leur professeur de français et une personne native, d'acquérir plus d'aisance à l'oral, d'avoir un peu plus de mots pour développer leur pensée analytique, d'améliorer aussi leur compréhension orale en français.

Selon notre avis, nous pourrions employer ce réseau comme un outil pédagogique dans le cours de Français Langue Étrangère de l'Universidad del Atlántico puisque cette université ne dispose pas d'un laboratoire de Langues pour que les étudiants fassent des activités de production et compréhension orale.

\section{References}

Arrivé, M., Gadet, F. \& Galmiche, M. (1986). La Grammaire d'aujourd'hui, Guide Alphabétique de Linguistique Française. Paris, France: Librairie Flammarion.

Besse, H. \& Porquier, R. (1991). Grammaire et Didactique des Langues. Paris, France: Hatier-Crédif.

Carnegie, D. (2014). Comment parler en public. Paris, France: Le Livre de Poche.

Corder, S. P. (1980). Que signifient les erreurs des apprenants? doi: 10.3406/lgge.1980.1833

Díaz, M. \& Gómez, S. (2013). L’apprentissage de langues étrangères: vers une culture
2.0. Revista de Investigación en Lenguas Extranjeras, 10(2), 117-137.

Larousse, P. (1995). Le Petit Larousse Illustré. [versión electrónica]. Paris, Fr: http:// www.larousse.fr/dictionnair

Le Glossaire Universel de la Psychologie et des Sciences Cognitives (2011). Le Glossaire Universel de la Psychologie et des Sciences Cognitives. Repéré à: http:// www.definitions-de-psychologie.com/fr/8.html

Menjura, M. (2007). La fluidez discursiva oral. Una propuesta discursiva, en Ogigia. Revista Electrónica de Estudios Hispánicos, (1), 8 .

Ministère de la Communication Niger (2003). Politiques et Stratégies pour le développement. Repéré à: ftp://ftp.fao.org/docrep/ fao/006/Y4957F/Y4957F00.pdf

Rosario, J. (2005). La tecnología de la información y la comunicación (TIC). Su uso como herramienta para el fortalecimiento y el desarrollo de la educación virtual. Repéré à: http://www.cibersociedad.net/ archivo/articulo.php?art=218

Tagliante, Ch. (1994). La classe de langue. Paris, France: CLE International, Coll. Techniques de Classe.

Vogel, K. (1995). L'interlangue, la langue de l'apprenant, traduit de l'allemand par Jean-Michel Brochee et Jean-Paul Confais. Toulouse, France: PUM. 
Tableau 1. Activité Diagnostique

\begin{tabular}{|c|l|c|c|}
\hline & \multicolumn{1}{|c|}{ Noms Et Prénoms } & Nombre de Syllabes en Français & Pourcentage du Nombre de Syllabes \\
\hline 1 & Baños Aguilar Melissa & 149 & $55,4 \%$ \\
\hline 2 & Benedetty Guriérrez Adriana Lucía & 152 & $56,5 \%$ \\
\hline 3 & Bustos Brochero Jean Michael & 105 & $39,0 \%$ \\
\hline 4 & Caldera Guerrero Katherine Esther & 69 & $25,7 \%$ \\
\hline 5 & Cervantes Sánchez Linda Stefany & 73 & $27,1 \%$ \\
\hline 6 & De La Ossa Arrieta María Angélica & 131 & $48,7 \%$ \\
\hline 7 & Meléndez Santander Carmen Alicia & 124 & $46,1 \%$ \\
\hline 8 & Molina De La Hoz Luis Miguel & 125 & $46,5 \%$ \\
\hline 9 & Navarro De Alba Lisbeth Patricia & 89 & $33,1 \%$ \\
\hline 10 & Ortega Caro Vanessa Paola & 117 & $43,5 \%$ \\
\hline 11 & Peña Ujueta Angélica & 151 & $56,1 \%$ \\
\hline 12 & Soñett Cañón Evaristo Antonio & 129 & $48,0 \%$ \\
\hline 13 & Urruchurto González Julieth Isabel & 55 & $20,4 \%$ \\
\hline 14 & Vergara Puello Saray Paola & 98 & $36,4 \%$ \\
\hline 15 & Zabaleta Pérez Jael & 119 & $44,2 \%$ \\
\hline & & & $100 \%$ \\
\hline & Locuteur Natif & 269 & \\
\hline
\end{tabular}

Source: Compiled by author

Tableau 2. Activité Finale

\begin{tabular}{|c|l|c|c|}
\hline & \multicolumn{1}{|c|}{ Noms Et Prénoms } & Nombre de Syllabes en Français & Pourcentage du Nombre de Syllabes \\
\hline 1 & Baños Aguilar Melissa & 238 & $88,5 \%$ \\
\hline 2 & Benedetty Guriérrez Adriana Lucía & 166 & $61,7 \%$ \\
\hline 3 & Bustos Brochero Jean Michael & 162 & $60,2 \%$ \\
\hline 4 & Caldera Guerrero Katherine Esther & 144 & $53,5 \%$ \\
\hline 5 & Cervantes Sánchez Linda Stefany & 153 & $56,9 \%$ \\
\hline 6 & De La Ossa Arrieta María Angélica & 199 & $74,0 \%$ \\
\hline 7 & Meléndez Santander Carmen Alicia & 239 & $88,8 \%$ \\
\hline 8 & Molina De La Hoz Luis Miguel & 156 & $58,0 \%$ \\
\hline 9 & Navarro De Alba Lisbeth Patricia & 156 & $58,0 \%$ \\
\hline 10 & Ortega Caro Vanessa Paola & 152 & $56,5 \%$ \\
\hline 11 & Peña Ujueta Angélica & 177 & $65,8 \%$ \\
\hline 12 & Soñett Cañón Evaristo Antonio & 190 & $70,6 \%$ \\
\hline 13 & Urruchurto González Julieth Isabel & 195 & $72,5 \%$ \\
\hline 14 & Vergara Puello Saray Paola & 152 & $56,5 \%$ \\
\hline 15 & Zabaleta Pérez Jael & 133 & $49,4 \%$ \\
\hline & & & $100 \%$ \\
\hline & Locuteur Natif & 269 & \\
\hline
\end{tabular}

Source: Compiled by author 
Tableau 3. Comparaison du pourcentage d'amélioration

\begin{tabular}{|c|l|c|c|c|}
\hline & \multicolumn{1}{|c|}{ Noms Et Prénoms } & $\begin{array}{c}\text { Pourcentage du Nombre } \\
\text { de Syllabes } \\
\text { Activité Diagnostique }\end{array}$ & $\begin{array}{c}\text { Pourcentage du Nombre } \\
\text { de Syllabes }\end{array}$ & $\begin{array}{c}\text { Activité Finale } \\
\text { Pourcentage } \\
\text { D'amélioration }\end{array}$ \\
\hline 1 & Baños Aguilar Melissa & $55,40 \%$ & $88,50 \%$ & $35,10 \%$ \\
\hline 2 & Benedetty Guriérrez Adriana Lucía & $56,50 \%$ & $61,70 \%$ & $5,20 \%$ \\
\hline 3 & Bustos Brochero Jean Michael & $39,00 \%$ & $60,20 \%$ & $21,20 \%$ \\
\hline 4 & Caldera Guerrero Katherine Esther & $25,70 \%$ & $53,50 \%$ & $27,90 \%$ \\
\hline 5 & Cervantes Sanches Linda Estefany & $27,10 \%$ & $56,90 \%$ & $29,70 \%$ \\
\hline 6 & De la Ossa Arrieta María Angélica & $48,70 \%$ & $74,00 \%$ & $25,30 \%$ \\
\hline 7 & Meléndez Santander Carmen Alicia & $46,10 \%$ & $88,80 \%$ & $42,80 \%$ \\
\hline 8 & Molina De la Hoz Luis Miguel & $46,50 \%$ & $58,00 \%$ & $11,50 \%$ \\
\hline 9 & Navarro De Alba Lisbeth Patricia & $33,10 \%$ & $58,00 \%$ & $24,90 \%$ \\
\hline 10 & Ortega Caro Vanessa Paola & $43,50 \%$ & $56,50 \%$ & $13,10 \%$ \\
\hline 11 & Peña Ujueta Angélica & $56,10 \%$ & $65,80 \%$ & $9,60 \%$ \\
\hline 12 & Soñétt Cañón Evaristo Antonio & $48,00 \%$ & $70,60 \%$ & $22,70 \%$ \\
\hline 13 & Urruchurto González Julieth Isabel & $20,40 \%$ & $72,50 \%$ & $52,00 \%$ \\
\hline 14 & Vergara Puello Saray Paola & $36,40 \%$ & $56,50 \%$ & $20,10 \%$ \\
\hline 15 & Zabaleta Pérez Jael & $44,20 \%$ & $49,40 \%$ & $5,20 \%$ \\
\hline & Locuteur Natif & $100 \%$ & $100 \%$ & \\
\hline
\end{tabular}

Source: Compiled by author

ANNEXE

Activité diagnostique. Documents Audio

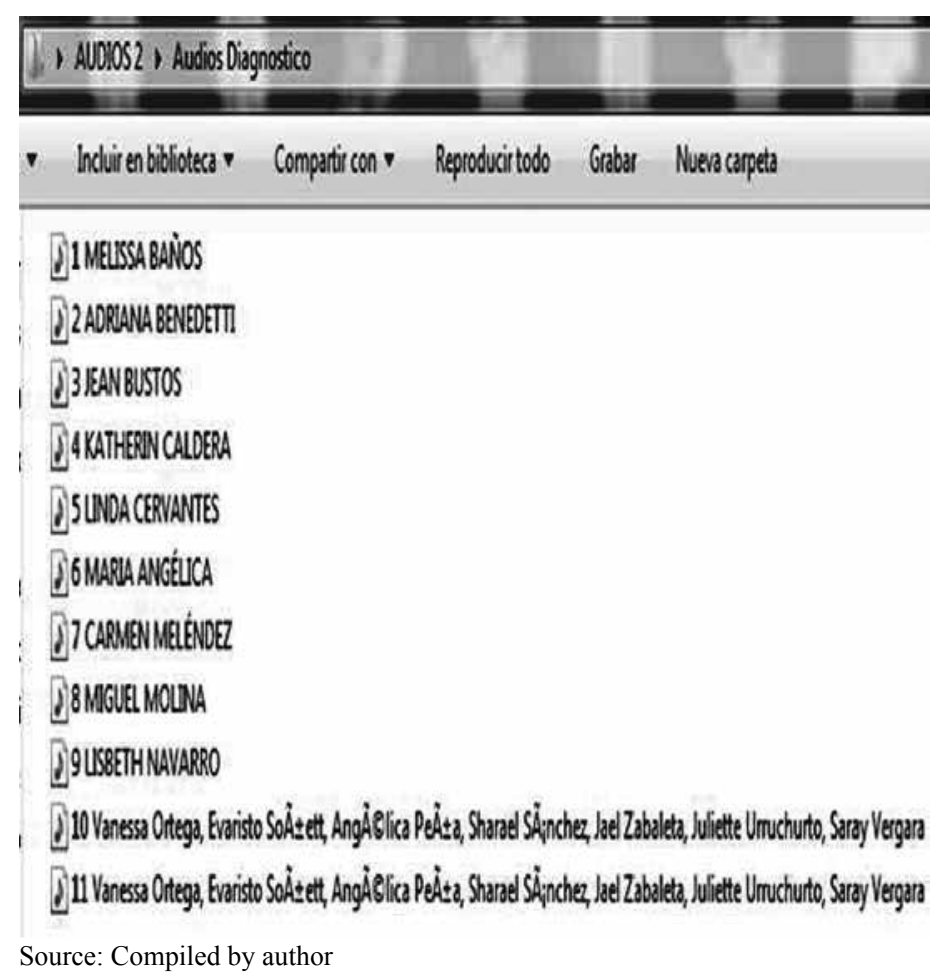


ANNEXE

Activités téléchargées sur Blaving

\section{questions du texte: Lucas sur la route}

dedycervantescerra.

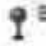

SOt5 - 2\$101 2000

ffte 0

consianes, des activités récentes

derlycrercantescerca

q

$20+5-2605$ it 50

f

te corbeau et le renard

dedlyserxantescerca.

9 asieded

$20+5+2405+13: 23$

f $\in$ : o

Flash info SNCF

denlycercanteseserca

quaters

$20: 5-2205+724$

f

Santè: boissons eneroisantes.

dedvoervantescerca

9 esiceses

$5015-226517: 10$

f

Rhonétique [] Rartie 2

derlycerdantescerra

p acleasa

2015-2106 500 ?

\section{If $上$ O}

Rhonétique [j]Rartie 1

derlycercantescerta.

9 toresos

2015 - 2504 2005

\section{f t}

Source: Compiled by author 


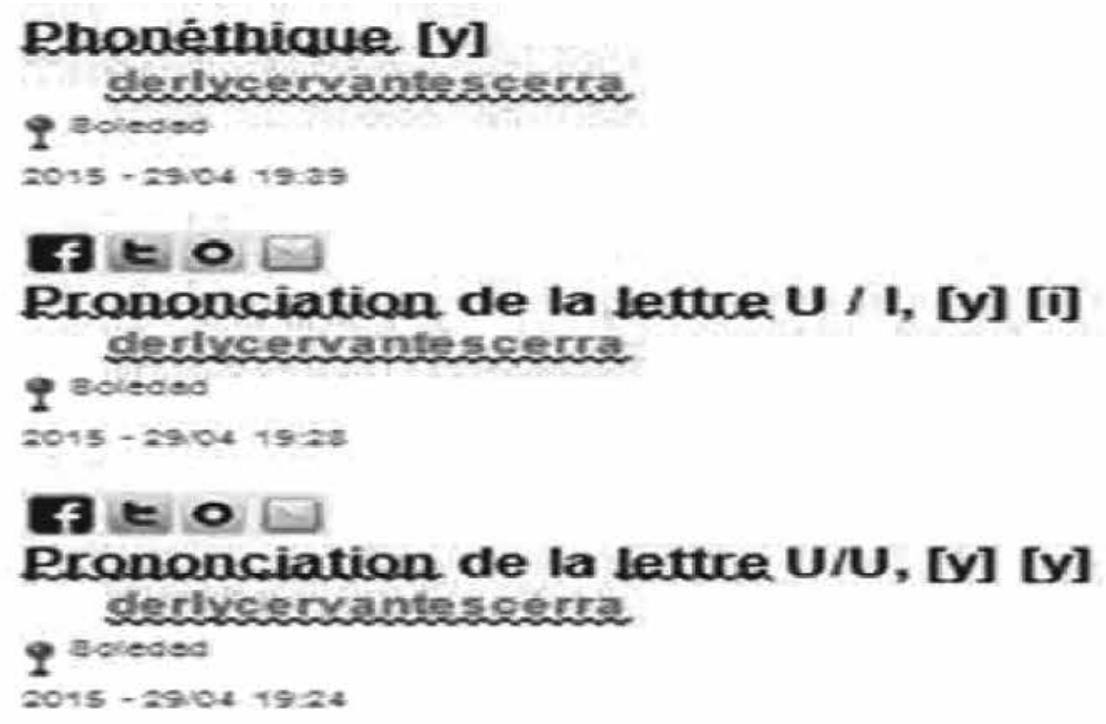

$\boldsymbol{F} \in \mathbf{0}$

Consignes des activités téléchargées

9 eoleded derlyoervantescerca

2015-0.:04,09:19

f $t=0 \Leftrightarrow$

Liaisons et Enchaînements derlyorisantescerra.

9 ableded

$2015-0.10417210$

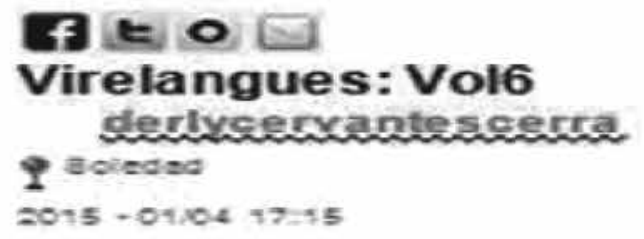

f it 0 D

Virelangues: Vol5

derlviervantescerra.

9

2015-0.104 1714

E $=0$

Source: Compiled by author 


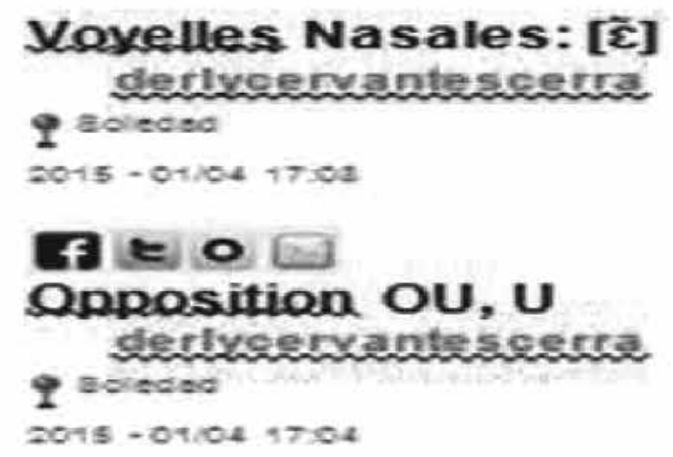

\section{f}

Opposition ON, AN

dertycercantesseves

9

2015 - 01i04 17.01

$f \in O \&$

Reportace: Entre nous

clertycervantescerca

9

-015-01104 15:05

$f \in O D$

REpontage: Portrait d'une princesse derixcervantescerra.

9 aclitded

$2015-01,04+15.02$

f

Reportage: pas facile la vie d'artiste

$\mathbf{9}$ derlyciervantescerra.

2015 - 01704 t5.00

$f=0$ G

Reportacepartir derlycervantesperra

9 eoledes

20.15-01,104 $14: 58$

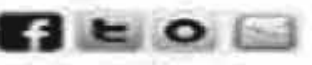


ANNEXE

Activité finale. Documents Audio

\begin{tabular}{|c|c|c|c|}
\hline 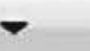 & Incluir en biblioteca & 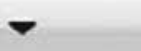 & Comparti \\
\hline \multicolumn{2}{|c|}{ Nombre } & Nú... & Título \\
\hline (2) & \multicolumn{3}{|l|}{ Adriana } \\
\hline D) & \multicolumn{3}{|l|}{ Angélica } \\
\hline D) & \multicolumn{3}{|l|}{ Carmen } \\
\hline D) & \multicolumn{3}{|l|}{ Evaristo } \\
\hline$D$ & \multicolumn{3}{|l|}{ Jael } \\
\hline D) & \multicolumn{3}{|l|}{ Jean M } \\
\hline D) & \multicolumn{3}{|l|}{ Juliette } \\
\hline D) & \multicolumn{3}{|l|}{ Katherin } \\
\hline$\$$ & \multicolumn{3}{|l|}{ Linda } \\
\hline D) & \multicolumn{3}{|l|}{ Lisbeth } \\
\hline D) & \multicolumn{3}{|l|}{ Maria Angélica } \\
\hline (3) & \multicolumn{3}{|l|}{ Melissa } \\
\hline (3) & \multicolumn{3}{|l|}{ Miguel } \\
\hline (2) & \multicolumn{3}{|l|}{ Saray } \\
\hline 8 & \multicolumn{3}{|l|}{ Vanessa } \\
\hline
\end{tabular}

Source: Compiled by author 
ANNEXE

Enquête auprès des élèves

UAAdiesididad

FACULTAD DE CIENCIAS HUMANAS

PROGRAMA DE LICENCIATURA EN IDIOMAS EXTRANJEROS

ENCUESTA SOBRE LA INCIDENCIA DE LA RED SOCIAL BLAVING COMO HERRAMIENTA PARA

MEJORAR LA FUIDEZ Y LA PRODUCCIÓN ORAL. EN LL CLASE. DE FRANCES LENGUA

EXTRANJERA, EN LOS ESTUDIANTES DE VIII SEMESTRE GRUPO I3

PROFESORA: DERLY CERVANTES CERRA

FECHA: le a vin 2015

QUESTIONS:

1. Quelles autres activités auriez-vous voulu travailler sur le rescau social Blaving?

Den conkersatrons expontanees par groupe, ya Veut dire des dialoges qi montrent linteraction entre plusieus, adiudus dan's in contexte réel

2. Qu' est-ce que l'utilisation du reseau Blaving vous a apponte ?

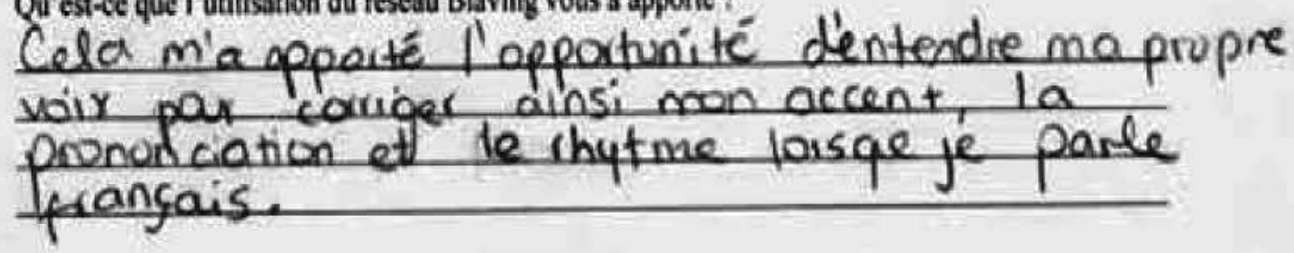

3. Croygrovous que cet outil aide à ameliorer la fluidité verbale des tléves?

a. (OUI) b. NON

Pourquoi?

Qui, car quand on fait lexercise d'essayer fusieuren fois in lexte (orall avant de venregistrer on arive a parler plus rapidement et nieux.

4. Croyez -vous qu' il aide it andliorer la prononciation des tleves?

(2001 b. NON

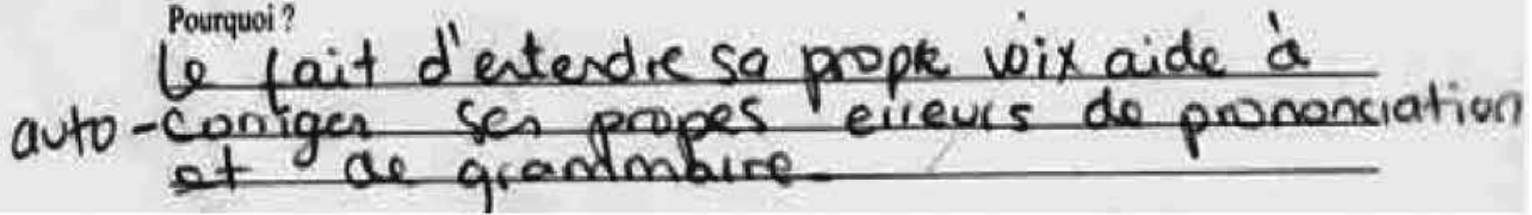

Source: Compiled by author 


\section{Universidad A del Atlántico IL mejor educiónal akance de todos!}

\section{FACULTAD DE CIENCIAS HUMANAS \\ PROGRAMA DE LICENCIATURA EN IDIOMAS EXTRANJEROS \\ ENCUESTA SOBRE LA INCIDENCIA DE LA RED SOCIAL BLAVING COMO HERRAMIENTA PARA MENORAR LA FUIDEZ Y LA PRODUCCION ORAL EN LA CLASE DE FRANCES LENGUA EXTRANERRA, EN LOS ESTUDIANTES DE VIII SEMESTRE GRUPO I3}

\section{PROFESORA: DERLY CERVANTES CERRA \\ FECHA: 1 JoW11 2015}

QUESTIONS:

1. Quelles autres activités auricz-vous voulu travailler sur le réseau social Blaving?

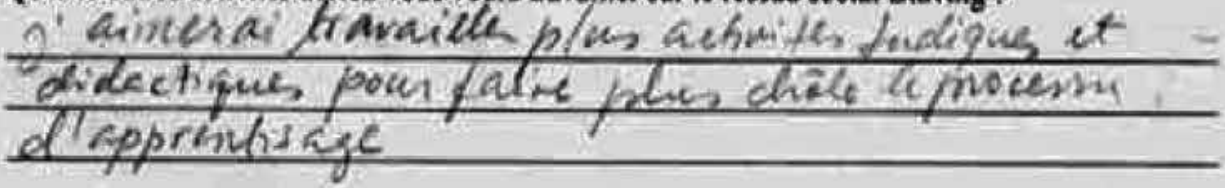

2. Qu'est-ce que l'utilisation du reseau Blaving vous a apponé?

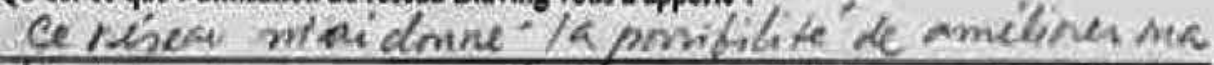

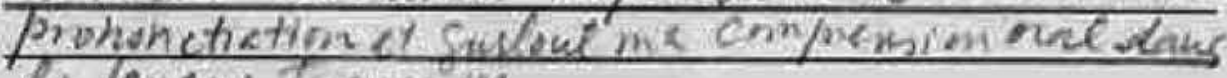 Le lengne Francerise.

3. Croyez-vous que cet outil aide da améliorer la fluidité verbale des élèves ?
(2.) OUI
b. NON

Pourquoi?

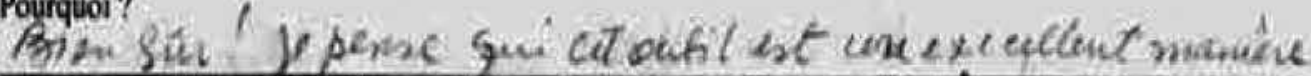

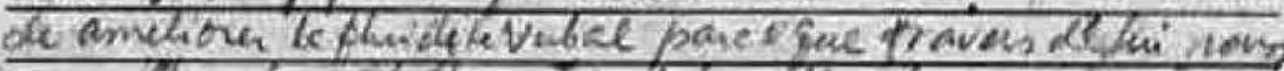

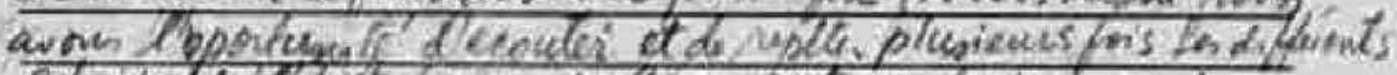

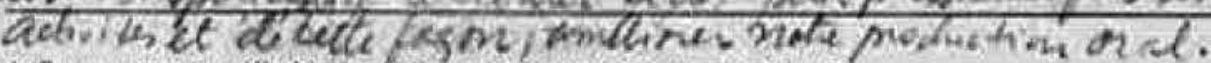

4. Croyez -vous qu'il aide á amelioner la prononciation des elétes?

\section{(aOUI b. NON}

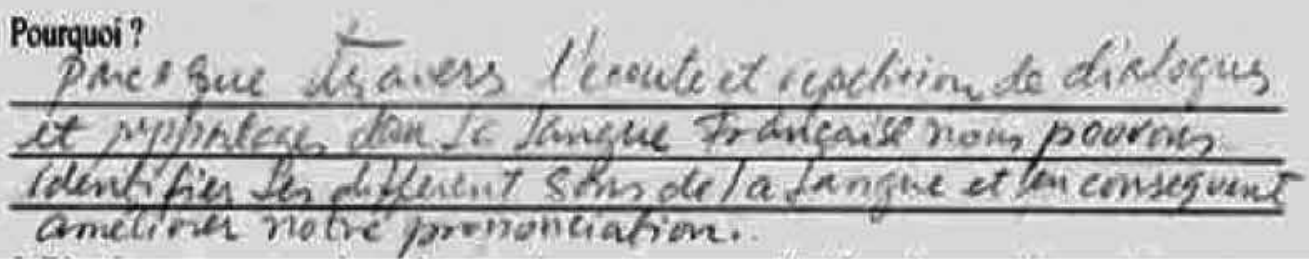

Source: Compiled by author 
ANNEXE

Entretien auprès des élèves. Documents Audio

\begin{tabular}{l} 
[1. ENTRETIE \\
\hline Incluir en \\
\hline Nombre \\
D. Entretien 5 \\
D. Entretien 4 \\
D. Entretien 3 \\
D. Entretien 2 \\
D) Entretien 1
\end{tabular}

Source: Compiled by author 